\title{
O MÁGico Jogo de AREIA EM PESQuisa ${ }^{1}$
}

\author{
Aicil Franco $^{2}$ \\ Elizabeth Batista Pinto ${ }^{3}$ \\ Instituto de Psicologia - USP
}

\begin{abstract}
Este trabalho apresenta o Jogo de Areia conforme concebido por Kalff (1980) numa abordagem teórica junguiana. Consideram-se alguns dos estudos que validam sua aplicação em serviços públicos de saúde, assim como com pacientes com câncer, vítimas de abusos e outros casos clínicos. A expansão clínica do Jogo de Areia como um método psicoterapêutico não verbal é também discutida. Considera-se e justificase, ainda, a necessidade do uso do Jogo de Areia como objeto de pesquisa. Também são apresentadas algumas possibilidades de ampliação do Jogo de Areia como um instrumento de pesquisa acadêmica na psicologia clínica, enfocando-se suas propriedades projetivas e diagnósticas.
\end{abstract}

Descritores: Jogo de areia. Psicologia junguiana. Pesquisa.

\section{O Jogo de Areia e sua fundamentação Junguiana}

$\mathrm{E}$ ste trabalho apresenta e discute o Jogo de Areia, método de psicoterapia junguiano, conforme concebido por sua autora (Kalff, 1980), enfocando aspectos do seu estágio atual de desenvolvimento no contexto de pesquisa em ps icologia clínica e sugerindo possibilidades de expansão.

1 Este trabalho é parte de pesquisa de dissertação de mestrado, em andamento, no curso de Pós-Graduação em Psicologia Clínica do IPUSP (julho de 2002).

2 Psicóloga, aluna do curso de Pós-Graduação em Psicologia Clínica do IPUSP. Endereço eletrônico: aicil@uol.com.br

3 Professora Doutora do Programa de Pós-Graduação em Psicologia Clínica do IPUSP. Endereço eletrônico: ebatista@usp.br 
O Jogo de Areia, como "método psicoterapêutico", foi idealizado por Dora Maria Kalff, analista junguiana suíça, a partir de técnica psicológica criada por Margaret Lowenfeld, pediatra inglesa que contemporaneamente à Melanie Klein introduziu o brinquedo na relação analítica com crianças (Thompson, 1981).

O procedimento criado por Kalff, entre 1954 e 1956, consiste na utilização de duas caixas preenchidas com areia e uma ampla coleção de miniaturas representativas de todo o universo (animais, pessoas, moradias, meios de transportes, vegetação, figuras mitológicas, religiosas etc.). O cliente, criança ou adulto, é convidado a escolher uma das caixas na qual deverá construir, com miniaturas à sua escolha, um cenário. A diferença entre as caixas é que, em uma delas, o cliente poderá acrescentar água à vontade, trabalhando de forma mais lúdica, emocionalmente mais regredida e com mais plasticidade. Pela padronização proposta por sua criadora (Kalff, 1980), é essencial que seja dada ao cliente a opção de duas caixas, especialmente porque a inchsão ou não de água na elaboração do cenário poderá ter características simbólicas importantes no processo. Kalff (1980) assumiu que a areia, de forma geral, por si só já é lúdica e convidaria à modelagem de formas ou cenas.

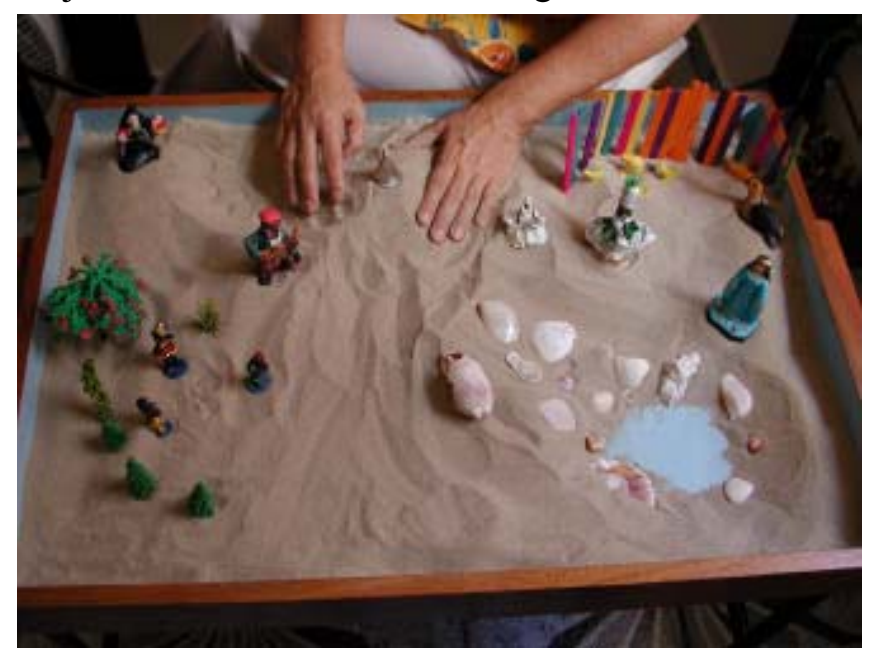

Figura 1: Caixa de areia. 


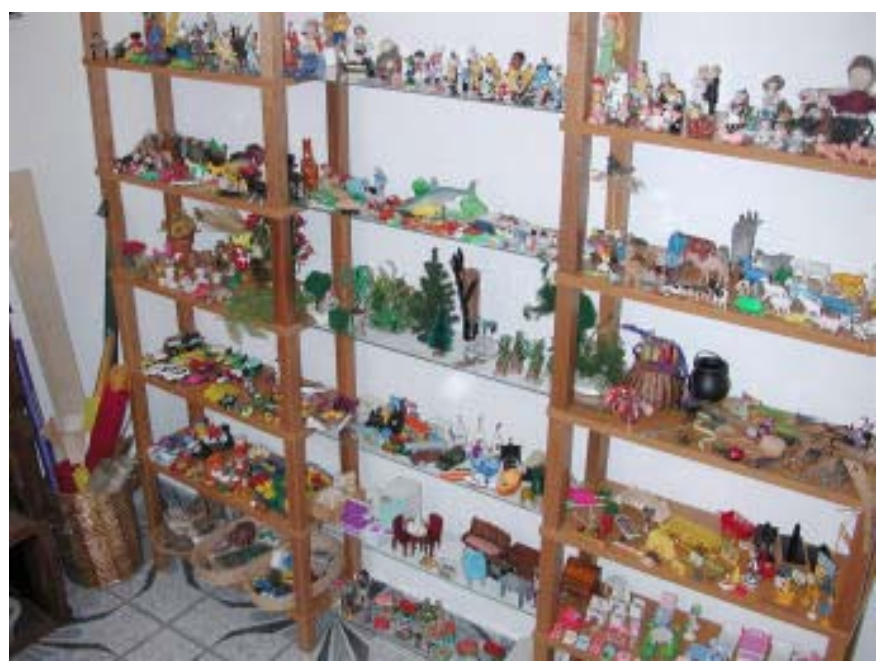

Figura 2: Miniaturas.

As caixas são padronizadas em forma (retangular, com $7,5 \mathrm{~cm}$ de profundidade), cor (internamente forrada de azul, favorecendo a fantasia do uso de seu fundo como água) e tamanho ( $72 \mathrm{~cm}$ x $50 \mathrm{~cm}$ aproximadamente), o qual corresponderia ao campo de visão de uma pessoa sentada na frente da caixa (Ammann, 1991). (veja figuras 1 e 2, caixa de areia e miniaturas, respectivamente).

O psicoterapeuta observará a criação do cliente, mas não intervirá. Segundo Kalff (1980) e Weinrib (1983), decidiu adaptar à sua criação o procedimento junguiano para interpretação de sonhos, o que significaria adiar, no processo de execução, as interpretações dos cenários.

O cenário permanecerá montado na caixa e será fotografado pelo psicoterapeuta depois da sessão, sem a presença do cliente. Após uma seqüência de cenários construídos - independentemente do tempo passado entre eles ou da utilização de sessões verbais intermediárias - cliente e terapeuta observarão juntos a série de fotografias correspondentes.

Nesse momento, o terapeuta poderá ou não fazer interpretações, sendo que diferentes autores que sucederam Kalff propuseram procedimentos di- 


\section{Aicil Franco e Elizabeth Batista Pinto}

versos. Observa-se que tais diferenças originam-se provavelmente na própria proposta da autora (Kalff, 1980), que não clarificou o que considerou por "adiar (ou suprimir) as inter pretações".

Alguns admitem, apenas circunstancialmente, a possibilidade de interpretações ao reverem as fotos (Weinrib, 1983, citado por Bradway, 1997); outros, ao contrário, consideram que permitir que o cliente saia da sessão sem uma interpretação imediata poderá representar um risco (RyceMenuhin, 1992, citado por Bradway, 1997); há, ainda, aqueles que advoguem a tese de que os insights do cliente serão sempre mais importantes e deverão se sobrepor, tornando desnecessária qualquer interpretação do terapeuta (Bradway, 1997).

A opinião de que o Jogo de Areia dispensaria qualquer interpretação baseia-se na existência de um suposto malefício representado pela interrupção do processo criativo, pressupondo que este seria terapêutico por si só: "É a vivência do processo, e não sua interpretação teórica, que cura" (Bradway, 1997, p. 46). Ou, ainda segundo Weinrib (1983), a caixa de areia por si só aceleraria e aprofundaria o processo psicoterapêutico.

Talvez seja importante distinguir o que seria uma interrupção do processo criativo por considerações cognitivas feitas pelo terapeuta durante a execução do cenário e o que seria uma interpretação que possibilitaria elaborações.

Partindo-se do pressuposto de que o Jogo de Areia é um procedimento inserido na relação clínica dual e transferencial, entende-se por interpretação dos cenários a investigação, por parte do terapeuta, da linguagem simbólica expressa pelo cliente, de forma que este possa conscientizar-se dos conteúdos surgidos nesse procedimento.

O terapeuta experiente fará essa investigação através da cuidadosa dbservação dos conteúdos apresentados nos cenários, da pesquisa de seu simbolismo e da análise de suas reações contratransferenciais, de forma a acumular possíveis sentidos para os conteúdos inconscientes, investigá-los e/ou interpretá-los, com o objetivo de estruturar ou ampliar a consciência do cliente. 
Ademais, no contexto da relação analítica, é genuíno pensar-se que intervenções acontecem consciente ou inconscientemente. O próprio Jung (1966) dedicou amplo estudo sobre a complexidade das projeções e transferências presentes nessa, e em outras relações. Conforme admitiu Jacoby (1995), todo o diagnóstico, assim como todas as interpretações e avaliações do material trazido pelo cliente, são baseados nos sentimentos e nas percepções do terapeuta.

Para ilustrar a configuração de transferência e de contratransferência que, tipicamente, acontece no Jogo de Areia, pode-se adequar o diagrama sobre a complexidade da relação analítica proposto, primeiramente, por Jung (1966) e, posteriormente, adaptado por Jacoby (1995) (veja figura 3). Percebe-se, nessa ilustração, que, no campo proposto como o da inconsciência comum, ou seja, o campo da interação entre paciente e terapeuta, incluem-se a caixa de areia e os cenários nela elaborados pelo cliente. Pode-se entender os cenários como produto da relação e, portanto, portadores, ainda que nconscientes, ou não verbais, também dos sentimentos, avaliações e intervenções do terapeuta.

P.

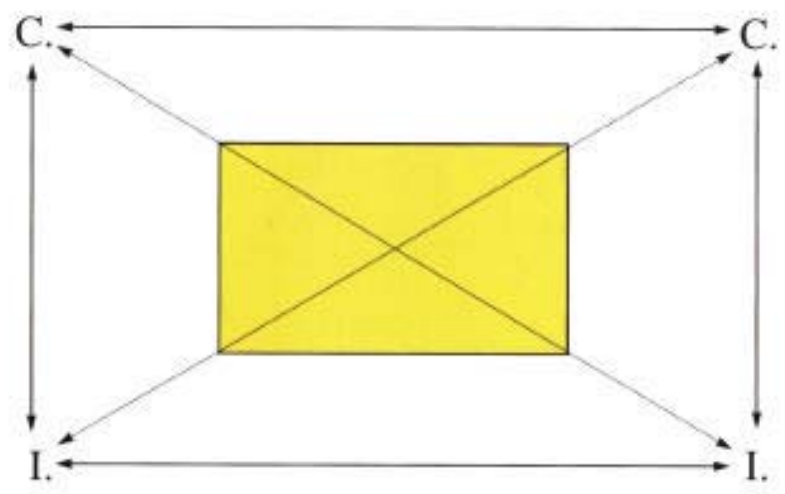

A.

Figura 3: Análise Junguiana. 


\section{Aicil Franco e Elizabeth Batista Pinto}

Kalff (1980) definiu, assim, o Jogo de Areia: ele "não é apenas um método de terapia, mas um meio ativo através do qual os conteúdos da imaginação são feitos reais e visíveis". Assim, alguns aspectos da situação psíquica poderiam ser projetados na caixa e vistos numa representação tridimensional. Essa transposição de conflitos do mundo interior para o mundo exterior os tornaria visíveis, e eles seriam, então, elaborados - pelo criador dos cenários - de maneira espontânea e evolutiva, "tal como o desenrolar de um drama".

O método de Kalff foi desenvolvido apoiando-se no pressuposto de que, quando o cliente começa a brincar ou construir cenários em intervalos regulares, poder-se-ia observar, também, o início de um processo de desenvolvimento psicológico, guiado por uma totalidade inconsciente. Jung (1991) reconheceu a existência desta totalidade inconsciente que, como um princípio de auto-regulação, tenderia a conduzir à união de aspectos contraditórios da personalidade que impedem o equilíbrio e o bem-estar psíquico. A expressão e a visualização, na caixa, dos aspectos conflituosos e de fontes de angústia levariam a psique, então, a acionar este princípio, que a orientaria na direção de um rearranjo mais harmonioso. A singularidade do Jogo de Areia seria, portanto, segundo sua autora, a de ser ele, por si mesmo, um mediador entre polaridades visíveis e invisíveis, conscientes e inconscientes (Kalff, 1980).

Entretanto, a partir da própria prática clínica e das conseqüentes e diferentes proposições dos autores, acima referidos, percebe-se a possibilidade de expandir a compreensão do Jogo de Areia se forem reconhecidas, nele, outras especificidades, como, por exemplo, sua capacidade como procedimento projetivo. Ora, a definição de um procedimento projetivo traz, em si, a necessidade de explicitação de critérios de interpretação. O indivíduo é posto na presença de situações pouco estruturadas e de estímulos ambíguos, os quais permitem "ler, segundo regras de decifração próprias do tipo de material e de atividade criadora propostos, certos traços do seu caráter e certos sistemas de organização do seu comportamento e das suas emoções" (Anzieu, 1960, citado por Laplanche \& Pontalis, 1989). 


\section{O Jogo de Areia e sua expansão clínica}

Desde sua concepção, no final de década de 50, o Jogo de Areia vem sendo divulgado e desenvolvido por meio da publicação de estudos em diversos países do mundo, especialmente EUA e Japão. Estes estudos vêm descrevendo um conjunto de procedimentos ou de técnicas que objetivam demonstrar a vantagem de seu uso em diferentes processos psicoterapêuticos, bem como discutir performances em determinados quadros patológicos.

Tais estudos consistem, principalmente, da descrição de casos clínicos, ou de análises dos símbolos presentes nos diferentes processos clínicos. Como, por exemplo: o processo de individuação feminina (Bradway, 1981), a transferência no Jogo de Areia (Kawai, 1985), as produções de alcoólicos (Kusas \& Hondas, 1990) e o acompanhamento de adolescente com câncer (Yosgikawa, 1999). Registram-se, ainda, estudos normativos ou comparativos que investigam tipos de construções de cenários feitos por certos grupos de pessoas, procurando correlações que levem ao estabelecimento de padrões de referências, como: Crable (1976), Denkers (1985), Grubbs (1995), Jones (1986), Mitchell e Friedman (1998) e Shaia (1991).

O uso do Jogo de Areia em serviços públicos de saúde já é discutido e aplicado, como ocorre, por exemplo, na Itália (Montecchi, 1993), em serviços de proteção aos abusos na infância, em hospitais para crianças com câncer e em instituições psiquiátricas.

Entretanto, a comunidade usuária e produtora de conhecimento sobre o Jogo de Areia tem demonstrado, ao longo dos últimos anos, preocupação com relação ao seu estágio de desenvolvimento no âmbito cientifico da psicologia clínica, conforme afirmou Ammann (1997):

\footnotetext{
Acredito que a terapia com o Jogo de Areia ainda não é completamente aceita e apreciada no campo da psicoterapia, e fazem-se necessárias mais pesquisas, juntamente com mais esforços de todos nós. Ainda permanecemos vistos como os "alquimistas" na psicoterapia, uma percepção que é agradável e de certa forma correta, mas isto também significa que permanecemos vistos como alquimistas misteriosos trabalhando de forma "não científica", "obscura" e miraculosa ... (p. 11)
} 


\section{Aicil Franco e Elizabeth Batista Pinto}

Em 1998, a própria International Society of Sandplay Therapy (ISST) criou uma comissão de pesquisa com objetivos de incentivar, principalmente, pesquisas científicas internacionais sobre o Jogo de Areia utilizado como método em "processos psicoterapêuticos junguianos" (Ammann, 1999).

Ainda não se conhece o resultado dessa comissão, embora observa-se que o engajamento na pesquisa acadêmica sobre o Jogo de Areia, em âmbito internacional, é pequeno e, até o momento, pouco contribuiu para as demandas da Psicologia. Nesse momento histórico da Psicologia como ciência, conforme afirmou Batista Pinto (1999), importaria que a pesquisa com o Jogo de Areia pudesse se afinar com a grande parte dos esforços que apontam para a necessidade de integração entre os interesses acadêmicos e a prática clínica.

No Brasil, a amplitude de interesses sociais requer da Psicologia clínica uma busca de formas de aplicação específicas, mais abrangentes e menos elitistas. A experiência com o Jogo de Areia permite especular acerca de sua possível adaptação a esta demanda. Além disso, de forma geral, no estágio de desenvolvimento atual da Psicologia clínica como ciência, no qual ainda é necessário que se solidifiquem práticas de pesquisa qualitativas, o Jogo de Areia poderá vir a se afirmar como um procedimento metodológico paradigmático (Batista Pinto, 1999).

É fato que esse procedimento se encontra em crescente expansão e vem se afirmando como atraente instrumento psicológico da prática clínica brasileira. Algumas pesquisas acadêmicas têm sido apresentadas por psicólogos brasileiros em congressos nacionais e internacionais de Psicologia.

Com características e objetivos bastante específicos, o uso da caixa de areia nestes estudos difere daquele proposto por Kalff (1980) como método psicoterapêutico, mas amplia o âmbito de atuação do Jogo de Areia. Vieira (1998) utiliza-se da caixa de areia como material lúdico em estudo sobre a capacidade da criança em expressar sua subjetividade. Já Gimenez (1998) e Zimmermann (1996) apresentaram, respectivamente, a caixa de areia como técnica facilitadora de integração de processos simbólicos e como instrumento facilitador para a orientação profissional. 
Outros estudos brasileiros têm sido realizados por grupos isolados, como alunos da Faculdade de Psicologia da Universidade Mackenzie (Universidade Mackenzie, 2002), que pesquisam a possibilidade de adaptação do Jogo de Areia na clínica-escola. O grupo Proteja (Projetos e Estudos em Terapia com o Jogo de Areia), formado por psicólogas clínicas de São Paulo, pesquisou a significação da presença de figuras femininas tipicamente brasileiras - como Iemanjá, a mulata e Nossa Senhora Aparecida - nos cenários dos clientes (Proteja, 1999). Esse grupo promoveu, ainda, simpósio no qual a possibilidade de pesquisa com o Jogo de Areia foi pioneiramente apresentada no Brasil e expandida para além dos limites acadêmicos, buscando integrá-la à prática clínica e a outras modalidades psicoterapêuticas (Simpósio Natureza e Simbologia do Feminino, 1999).

\section{Pesquisa cientifica com e sobre o Jogo de Areia}

Ao se admitir a necessidade de que mais pesquisas sejam realizadas com o Jogo de Areia, deve-se levar em conta que interesses menores - como os de pequenos agrupamentos profissionais e de comunidades não científicas - não deveriam se sobrepor à diversidade de demandas internacionais e, principalmente, à especificidade das demandas nacionais, sendo, pois, fundamental, que sejam levadas em consideração distintas características dos contextos sociais e políticos em que essas pesquisas serão realizadas.

Ao mesmo tempo, é importante discriminar quais pesquisas seriam realizadas com o Jogo de Areia e quais seriam realizadas sobre o Jogo de Areia. Ou seja, é desejável que o Jogo de Areia, diferente do que vem sendo feito, também deveria se prestar a ser objeto de pesquisa.

Pesquisas com o Jogo de Areia têm enfatizado, predominantemente, suas eficientes propriedades terapêuticas, mas, em geral, se eximem de explicar fenômenos clínicos ou mecanismos psicológicos com um rigor me todológico que lhes ofereça credibilidade. Ainda que se possa observar, empiricamente, qualidades do Jogo de Areia, as quais lhe atribuem significativo valor psicoterapêutico, é necessário que, por meio de mais pesquisas cientí- 
ficas, consiga-se demonstrar sua importância como instrumento da psicologia clínica, retirando-o do arcabouço dogmático em que ainda se encontra. Tratar-se-ia, então, da investigação sobre o Jogo de Areia, sobretudo de seus aspectos teóricos e funcionais.

Nesse caso, caberia, por exemplo, aprofundar a discussão acerca da afirmação dos autores mencionados acima, segundo a qual a vivência do processo seria, por si mesma, curativa. Ou, então, explorar os possíveis aspe ctos da subjetividade implicados na relação entre cliente e terapeuta, os quais, presentes na "vivência do processo", fazem-no menos ou mais curativo. Ou mesmo desenvolver uma metodologia de investigação que especifique a validade do Jogo de Areia, com generalizações rigorosas ou transferibilidades partilháveis que pudessem tirá-lo do pejorativo lugar de "miraculoso" ou "mágico".

Recorde-se, aqui, que, em metodologia científica, entende-se que a generalização dos resultados, considerada critério de qualidade nas investigações quantitativas clássicas, equivale à noção de transferibilidade, adotada como critério de boa qualidade nas investigações qualitativas. Transferibilidade de resultados de um estudo é a possibilidade de utilização posterior em situações semelhantes - dos procedimentos e resultados validados em uma situação inicial, respeitando-se as peculiaridades de novos contextos (Valles, 1997, citado por Silva, 1998).

\section{Possibilidades de expansão de pesquisa científica com o Jogo de Areia}

No âmbito da pesquisa científica em Psicologia clínica, apresentam-se diversas questões sobre os aspectos intrínsecos da relação que se estabelece com o objeto de estudo. Ao se realizar um projeto de pesquisa sobre psicoterapias psicodinâmicas, deve-se especificar o fenômeno e a situação a serem estudados. A metodologia de investigação ou o método de pesquisa considera a subjetividade que envolve a relação entre sujeito e objeto.

Assim como outras abordagens psicoterapêuticas psicodinâmicas, a investigação na Psicologia junguiana permite que se adote uma abordagem 
qualitativa de pesquisa em que fenômenos ou fatos clínicos sejam descritos. Esta abordagem, por sua vez, permite que se discutam aspectos subjetivos da atividade clínica que tendem a se repetir em diferentes processos, preservando singularidades e idiossincrasias. Seu maior mérito está na contribuição que pode oferecer à construção do conhecimento da singularidade humana, permitindo que se aplique o critério de transferibilidade dos resultados.

Enfatiza-se, assim, o mérito do Jogo de Areia como um procedimento qualitativo por excelência, no qual características psíquicas podem ser visualizadas e descritas de forma absolutamente singular e passível de transferibilidade. Suas evidentes especificidades psicológicas permitem supor que a ampliação de seu estudo, efetuada também por outras abordagens teóricas, só traria vantagens para a Psicologia como ciência e para a elucidação da eficácia do método.

Considerando-se essas especificidades, como se verá em Especificidades psicológicas do Jogo de Areia, enfatiza-se nesse trabalho a importância da inserção do Jogo de Areia no universo dos procedimentos projetivos, cuja teoria geral não necessariamente se atém a um único enfoque epistemológico. Não se trata de retirar os méritos da abordagem junguiana do método, mas sim, de encarar a tarefa de explorar toda a riqueza inerente ao procedimento, em benefício do conhecimento científico.

Assim, o desafio da realização de pesquisa científica com o Jogo de Areia não terá, a priori, de se manter circunscrito apenas ao quadro teórico da Psicologia Analítica, mas poderá recorrer a aportes de outras filiações teóricas e epistemológicas. Tanto a pesquisa com quanto a pesquisa sobre o Jogo de Areia poderão, portanto, partir de diferentes enfoques e adotar princípios epistemológicos de diversas teorias - sem descuidar, evidentemente, dos perigos de um ecletismo teórico que, na maioria das vezes, acaba por descaracterizar o próprio objeto de pesquisa. Considerando-se, pois, a subjetividade inerente à tarefa, importará que o rigor do método de investigação, da descrição dos critérios e dos fenômenos seja coerente com os princípios teóricos adotados. 


\section{Aicil Franco e Elizabeth Batista Pinto}

\section{Especificidades psicológicas do Jogo de Areia}

\section{A - Qualidades projetivas}

Embora pouco se encontre na bibliografia especializada informações sobre as propriedades projetivas do Jogo de Areia, estas características são também reconhecidas, como em Weinrib (1983, p. 78): "Ao externar sua fantasia, ele (o paciente) a projeta nas miniaturas". No âmbito da psicologia clínica, a propriedade projetiva do Jogo de Areia, talvez, seja sua melhor contribuição para a compreensão e validação do método.

O conhecimento acumulado sobre procedimentos projetivos e a experiência com o Jogo de Areia permitem que se amplie a compreensão da definição do procedimento criado por Kalff (1980) e que se coloquem as propriedades do Jogo de Areia em posição similar à das técnicas de investigação clínica, conforme definidas por Trinca (1997). Segundo aquele autor, as técnicas de investigação clínica oferecem acesso não apenas a dhdos relativos a um momento psicológico do indivíduo, mas também registram características do seu desenvolvimento emocional e da qualidade de relação entre cliente e psicoterapeuta.

Sabe-se que os instrumentos projetivos de psicodiagnóstico podem revelar aspectos que o paciente não traz para o contexto de psicoterapia, omissão que, às vezes, decorre de um pacto inconsciente com o psicoterapeuta. Instrumentos projetivos podem revelar sofrimentos profundos que ficam ausentes da área mental de consciência, sendo, pois, um procedimerto a mais à disposição da clínica psicológica (Grassano, 1996).

Assim, os cenários na caixa de areia - na observação de sua elaboração e em seu produto final - permitem ao psicólogo experiente inferir sobre aspectos mais amplos do desenvolvimento psíquico do cliente, sendo possível levantar hipóteses para além das características de personalidade e do momento terapêutico vivenciado.

Esse aspecto, mais uma vez, coloca em discussão singularidades do Jogo de Areia, as quais permitem que ele seja considerado, ora como método psicoterapêutico (Kalff, 1980), ora como coadjuvante em proces- 
sos psicoterapêuticos, ora como procedimento projetivo de investigação clínica, dependendo da contextualização de seu uso. Talvez se deva lembrar ainda, conforme afirmou Safra (1996) em relação à pratica psicanalítica, que clinicar é investigar. Também na abordagem junguiana e em outras psicoterapias psicodinâmicas, o trabalho clínico integra-se ao trabalho investigativo. Ou seja, a execução de cenários na caixa de areia pode ser vista, ao mesmo tempo, como um procedimento psicoterapêutico e investigativo.

A qualidade dos sentimentos (tristeza, alegria, raiva, hostilidade, ins egurança etc.), as principais defesas (negação, sublimação, regressão etc.), as tendências dos impulsos (obs essão, perfeccionismo, excitação, espontaneidade, contenção etc.), os diferentes aspectos de desenvolvimento intelectivo (falta de concentração, limitação, alta elaboração, abstração etc.), as funções psicomotoras (uso do espaço, esquema corporal, lateralidade, equilíbrio, coordenação viso-motora etc.) e mesmo os indícios de comprometimentos neurológicos podem ser perceptíveis por meio dos gestos, da manipulação das miniaturas, da elaboração das cenas e da relação destes - gestos, miniaturas e cenas - com o espaço das caixas. Aponta-se, aqui, a inexistência de pesquisas nessa área, as quais, por exemplo, poderiam fornecer material para compreensão de alguns distúrbios psiconeurológicos, ainda hoje de difícil avaliação (por exemplo, toda a complexidade das ga gueiras e das afasias).

Entende-se que o Jogo de Areia, na qualidade de um jogo simbólico ou como técnica de investigação clínica, possa ser considerado um poderoso instrumento projetivo, que possibilita a conscientização e interpretação de conteúdos que a inda não podem ser totalmente expressos em palavras e que, em muitos casos, dificultam o diagnóstico ou o direcionamento de um tratamento multidisciplinar. A atividade lúdica, aparentemente desvinculada da queixa, pode diminuir a ansiedade do cliente, que se sente menos ameaçado para se expressar de forma que parece ser controlada apenas por ele próprio.

O Jogo de Areia como procedimento projetivo pode ser ilustrado por um caso clínico no qual havia uma suspeita inicial de autismo, com difícil elucidação diagnóstica. A cliente mostrava-se resistente a qualquer tentativa de interação, mesmo nas situações lúdicas. Manifestava, dentre outros, com- 


\section{Aicil Franco e Elizabeth Batista Pinto}

portamentos de isolamento e traços típicos de neurose obsessiva, com sérias conseqüências sociais. A introdução do Jogo de Areia, aplicado de forma bastante flexível e alternativa às propostas originais de Kalff (1980), permitiu, inicialmente, gradativas mudanças no comportamento da cliente nas sessões terapêuticas e, posteriormente, a compreensão de aspectos psicológicos familiares graves, expressos nos cenários, aos quais não se teve acesso por outros procedimentos. Observou-se, a partir de então, a crescente interação da cliente, não apenas com a terapeuta, mas também com as demais pessoas de seu relacionamento. Os cenários do Jogo de Areia permitiram diferenciações diagnósticas, visualização do desenvolvimento intelectual e da personalidade, explicações da dinâmica do caso, encaminhamentos adequados e orientações a familiares e professores (Araújo \& Franco, 1998).

Esse estudo ilustra o potencial projetivo do procedimento, aponta a eficácia de sua aplicação para tratamentos de casos, tidos, em geral, como refratários aos tratamentos psicoterapêuticos clássicos e reforça a necessidade de pesquisas aprofundadas e rigorosas que possam ser referência para diagnóstico e tratamento de diferentes psicopatologias.

\section{B - Qualidades psicodiagnósticas}

Kalff (1980) desenvolveu seu método a partir de pesquisas anteriores tanto de Lowenfeld (Mitchell \& Friedman, 1994), como de Bolgar e Fischer (1940) e também de Bühler (1951), que utilizavam o World Technique (nome original da técnica psicológica proposta por Lowenfeld), principalmente como instrumento de psicodiagnóstico. Essas pesquisas incluíam estudos de correlação e pretendiam testar a capacidade do instrumento para diagnosticar patologias, comparável ao Rorschach e outras técnicas projetivas reconhecidas (Mitchell \& Friedman, 1994, p. 115).

De fato, desde os seus primórdios, o Jogo de Areia já se caracterizava como um meio através do qual a vida emocional e mental das crianças era comunicada numa forma que poderia ser objetivamente registrada e analisada. Além disso, segundo Friedman e Mitchell (1994): 
Lowenfeld viu transferência nos "mundos" de seus clientes quando eles mostravam seus aspectos primários na caixa através da escolha das miniaturas, de seus arranjos espaciais e de suas histórias sobre as caixas de areia, mais do que percebeu transferência numa perspectiva tradicionalmente psicanalítica - somente entre terapeuta e cliente. Com a ajuda desta técnica, ela (Lowenfeld) poderia agora começar seu trabalho de exploração do processo mental da criança. (p. 79)

Kalff, entretanto, provavelmente influenciada por referências da prática clínica na Psicologia Analítica, foi enfatizando cada vez mais a aplicação do Jogo de Areia como método de expressão simbólica em contexto psicoterapêutico. Tanto Kalff como alguns de seus seguidores evitaram métodos quantitativos ou abordagens diagnósticas para a compreensão dos cenários (Mitchell \& Friedman, 1994, p. 87).

Embora o Jogo de Areia possa ser usado como instrumento de psicodiagnóstico, não é assim que é visto pela comunidade internacional usuária do método: "Não é um diagnóstico nem um teste psicológico, principalmente por ser encarado como um método que se desenvolve num processo psicodinâmico de inter-relação do paciente e terapeuta, ao longo do tempo, meses ou anos" (Ammann, 1999).

Percebe-se nas afirmações dessa autora (Ammann, 1999) e, em geral, nas referências presentes na bibliografia especializada, que o enquadre ps icoterapêutico junguiano parece rechaçar propriedades psicodiagnósticas nas características do Jogo de Areia. Segundo Mitchell e Friedman (1994):

... esta atitude reflete uma perspectiva junguiana básica, na qual a psique évista como tendo em si a capacidade de promover a totalidade, e não como, do ponto de vista médico, segundo o qual o comportamento é rotulado por uma autoridade externa, para fins de tratamento.

Entretanto, o Jogo de Areia talvez possa vir a se afirmar como mais um importante recurso, se, como propôs Trinca (1997), atribuirmos um novo status para o diagnóstico psicológico, por meio de uma abordagem mais fléxivel e de tipo mais compreensivo, na qual prevaleça antes o espírito de nvestigação do que a submissão aos padrões estabelecidos pelas teorias dominantes. 
Considerando-se as características descritas por Vilhena (1997, citada por Tardivo, 1997) como as necessárias para um psicodiagnóstico na abordagem junguiana, o Jogo de Areia já se aplicaria nessa especificidade da Psicologia clínica, uma vez que se trata de um instrumento que possibilita "a análise do desenvolvimento arquetípico-simbólico através da emergência de símbolos possivelmente constelados no eixo ego-Self'. Além disso, o Jogo de Areia possibilitaria o "levantamento de hipóteses sobre as capacidades defensivas e criativas, operantes e potenciais do cliente". Nos cenários do Jogo de Areia, pode-se também visualizar - conforme priorizou aquela autora (Vilhena, 1997, citada por Tardivo, 1997) - o "padrão transferencial e as elaborações criativas precoces".

A própria Kalff reconheceu, segundo Mitchell e Friedman (1994), a propriedade de cenários iniciais como facilitadores na compreensão da direção a ser impressa no tratamento. Após conferências de 1966, Kalff teria publicado um artigo no qual enfatizou que o primeiro cenário de uma série ofereceria ao terapeuta um guia para o tratamento, porque freqüentemente daria informações - envolvidas nos símbolos - sobre a natureza do problema, o prognóstico e como a cura iria ocorrer (Mitchell \& Friedman, 1994).

Montechi (1993) também observou e ilustrou como, numa fase preliminar do tratamento, os cenários na caixa de areia podem fornecer avaliações sobre a "tratabilidade ou não do caso (pelo menos com esse instrumento terapêutico)".

Aberastury (1982) constatou que "já durante a primeira sessão - fosse esta o início de uma análise ou simplesmente de observação diagnóstica aparecia fantasia inconsciente de enfermidade ou cura" (p. 111). A mesma autora concluiu que, em todos os casos de crianças estudados por ela, a criança sabia que estava enferma e comunicava, desde a primeira hora, qual era sua fantasia inconsciente sobre a enfermidade ou conflito pelo qual era trazida ao tratamento e, na maior parte dos casos, apresentava, também, sua fantasia inconsciente de cura (Aberastury, 1982).

Especialmente no que tange à possibilidade de investigação psicodiagnóstica do primeiro cenário do Jogo de Areia e ao seu caráter de investi- 
gação clínica da personalidade, pode-se considerar, como paradigmas de estudo, aportes teóricos como os de Aberastury (1982), devido aos aspectos comuns da relação e aos objetivos similares - investigação e tratamento psíquico.

As afirmações dessa autora também corroboram estudos feitos por autores especialistas no Jogo de Areia (Amatruda, 1998a; Amatruda, 1998b; Amatruda, 2000; Bradway, 1997; Franco, 1998; Macnofsky, 1996), segundo os quais os primeiros cenários, analisados retrospectivamente, mostraram, por exemplo, sinais evidentes de grave doença orgânica que, algumas vezes, se manifestariam anos depois. A hipótese de que, inconscientemente, a presença de uma doença física se apresenta nos cenários de clientes submetidos ao Jogo de Areia está freqüentemente sendo estudada e descrita por alg uns autores. Ramos (1999) afirmou que a psique contém informações às quais o consciente não tem acesso, mas que podem aparecer em sonhos e/ou em cenários na caixa de areia, revelando registros de doenças orgânicas e também dos símbolos que poderiam indicar elementos faltantes para o paciente ampliar a consciência de si mesmo e até mesmo curar-se.

Bradway (1992) apresentou estudos sobre o que chama de "cenário corporal", no qual corpos ou partes de corpos podem ser visualizados na configuração do cenário, feitos de forma inconsciente pelos clientes, ou seja, provavelmente revelando o conhecimento inconsciente de alguma anomalia ou tensão em relação ao corpo. Por exemplo, apareceram "montanhas" na areia que se configuraram como seios num corpo de mulher, ou "lagos" que podem apresentar formas semelhantes a pulmões ou rins.

Ramos (1999) corroborou esta idéia, afirmando que pacientes psicossomáticos têm, em geral, estruturação psicológica muito regressiva e vivenciam graves conflitos em um nível muito profundo no qual a dor emocional seria insuportável. A doença orgânica seria, nesses casos, símbolo de uma disfunção maior, provavelmente na relação ego-self. O Jogo de Areia utilizado com esses pacientes permitiria o aparecimento simbólico desse sofrimento e, provavelmente, sua constelação corpórea, numa forma em que o 
paciente poderia sentir-se protegido para a integração consciente do símbolo, e, provável, resolução do conflito.

Bradway (1994) também estudou os primeiros cenários de clientes, comparando o desenvolvimento, em psicoterapia, de dois grupos de mulheres, um dos quais chamou de "profissionalizadas" e outro de "mulheres do lar". Percebeu aspectos comuns e aspectos diferenciados inter e intragrupais que lhe possibilitaram várias generalizações, encorajando estudos que explorem as potencialidades de primeiros cenários no Jogo de Areia em outras pesquisas comparativas.

Outros pós-junguianos, como Hall (1983), também consideraram que um psicoterapeuta experiente pode-se utilizar de material onírico, e estenderam essa aplicação ao conteúdo simbólico do Jogo de Areia, entendendo-o como um fator adicional na avaliação diagnóstica e prognóstica, assim como sutil indicador da oportunidade de instituir ou alterar medicação, considerar hospitalização e/ou variar a freqüência das sessões terapêuticas.

O mesmo autor (Hall, 1983) compreendeu os sonhos como elementos de observação acerca do funcionamento intelectual do cliente e de sua capacidade para sintetizar, de sua orientação quanto ao tempo, lugar e situação. Outros aspectos subjetivos de importância diagnóstica, presentes em conteúdos oníricos e que se assemelham aos conteúdos simbólicos do Jogo de Areia, referem-se a dimensões afetivas e emocionais, bem como a sugestões de desfechos para questões específicas.

Assim, a despeito da desconsideração de alguns autores quanto ao valor diagnóstico e quanto à necessidade de que se explicitem critérios de interpretação que possam complementar a eficácia do Jogo de Areia e até estabelecer padrões de referência para diferentes graus e quadros de psicopatologia, percebe-se que sua possibilidade de contribuição nessa área da psicologia clínica merece mais estudos.

Pode-se supor que o Jogo de Areia permitiria avaliações significativas e interpretações baseadas em diferentes referenciais teóricos, o que certamente não o equipararia a testes psicológicos - os quais têm sido vistos com preconceituoso demérito -, mas provavelmente daria conta de outro modo de 
investigação da personalidade, mais flexível e de tipo mais compreensivo, como afirmou Trinca (1997) acerca de outras técnicas de investigação clínica.

\section{Conclusões}

Partindo dos relatos sobre a eficiência do Jogo de Areia no contexto das psicoterapias, pode-se dizer que ele vem se afirmando como um método psicoterapêutico, desenvolvido com um instrumental clínico junguiano singular.

Reconhece-se a necessidade de ampliação do conhecimento existente sobre o Jogo de Areia, supondo-se que, através dele, a psicologia clínica possa ter alguns mecanismos psíquicos mais bem explicados, o que propiciaria articulações teóricas talvez ainda não estabelecidas e, ao mesmo tempo, retiraria o Jogo de Areia do lugar "mágico" em que ele ainda é injusta e insistentemente colocado.

O Jogo de Areia apresenta amplas possibilidades de estudo para o favorecimento psicoterapêutico de adultos e crianças em situações especiais, provavelmente prestando-se a diferentes abordagens teóricas.

De forma mais abrangente, o Jogo de Areia pode vir a se estabelecer, também, como rico método de pesquisa na psicologia clínica, necessitando de maior divulgação e pesquisa para sua solidificação e reconhecimento.

Especialmente no que tange à demanda brasileira e de outros países similares, deve-se considerar a possibilidade de a aplicação do Jogo de Areia ser inserida em pesquisas que respeitem as especificidades sócio-políticoculturais.

A demanda por atendimentos psicológicos no Brasil está longe de ser caracterizada e atendida. Portanto, deve-se perseguir formas nas quais os serviços psicológicos possam ser mais acessíveis, rápidos e eficazes; este parece ser um desafiante e imenso campo aberto para a intervenção e a pesquisa com o Jogo de Areia. 


\title{
Aicil Franco e Elizabeth Batista Pinto
}

Franco, A., \& Batista Pinto, E. (2003). The magic sandplay in research. Psicologia USP, 14(2), 91-114.

\begin{abstract}
This paper presents Sandplay as conceived by Kalff (1980) in a Junguian theoretical approach. We summon up some of the studies for the validation of its application in public health services as with patients suffering from cancer, abuse victims and other clinical patients. The clinical expansion of Sandplay as a nonverbal psychotherapeutic method is also discussed. The need for Sandplay use as a subject of scientific research is considered and justified. We also present some possibilities to enlarge the application of Sandplay, focusing its projective and diagnostic qualities, as an instrument for academic research in clinical psychology.
\end{abstract}

Index terms: Sandplay. Jungian psychology. Research.

Franco, A., \& Batista Pinto, E. (2003). La magie du jeu de sable en recherche. Psicologia USP, 14(2), 91-114.

Résumé: Ce travail présente le Jeu de Sable, celui conçu par Kalff (1980), dans un abordage théorique jungien. On prend en compte quelques-unes des études qui valident son application dans les services publics de santé ainsi que chez des patients cancéreux ou victimes d'abus, et d'autres cas cliniques. L'expansion clinique du Jeu de Sable comme méthode psychothérapique non verbale est aussi discutée. On étudie et on justifie, encore, la nécessité de l'usage du Jeu de Sable comme objet de recherche. On présente également quelques possibilités d'extension du Jeu de Sable comme instrument de recherche académique en psychologie clinique, en se concentrant sur ses propriétés projectives et de diagnostic.

Mots clés : Jeu de sable. Psychologie jungienne. Recherche.

\section{Referências}

Aberastury, A. (1982). A psicanálise da criança: Teoria e técnica. Porto Alegre, RS: Artes Médicas. 


\section{O Mágico Jogo de Areia em Pesquisa}

Amatruda, K. (1998a). Lecture in Hawaii. Trabalho apresentado em Conferências organizadas pela Sociedade Americana de Terapeutas de Jogo de Areia (STA). Texto não publicado.

Amatruda, K. (1998b). Sandplay process of a boy with cancer. Journal of Sandplay Therapy, STA, 7(2).

Amatruda, K. (2000). The haunted boy. Journal of Sandplay Therapy, STA, 9(1).

Ammann, R. (1991). Healing and transformation in sandplay. Illinois: Chicago Open Court and La Salle.

Ammann, R. (1997). Some reflections on the position of field of sandplay within the vast and difficult psychotherapeutic methods and professional demands. Trabalho apresentado no 14o Congresso da Sociedade Internacional de Terapia com Jogo de Areia, Verona, Itália.

Ammann, R. (1999). A pesquisa com o jogo de areia. Trabalho apresentado no Simpósio Natureza e Simbologia do Feminino: Ensaio, Pesquisa e Arte [Filmevídeo]. São Paulo: PROTEJA. 1 cassete VHS, 60 min. color. son.

Araújo, I. C. R., \& Franco, A. (1998). A eficácia do jogo de areia num caso de neurose obsessiva com suspeita de autismo. Trabalho apresentado no 3o Encontro da Sociedade Brasileira de Rorschach e Outros Métodos Projetivos, Ribeirão Preto, SP.

Batista Pinto, E. (1999). A singularidade da pesquisa em psicoterapia. Trabalho apresentado no Simpósio Natureza e Simbologia do Feminino: Ensaio, Pesquisa e Arte [Filme-vídeo]. São Paulo: PROTEJA. 1 cassete VHS, 60 min. color. son.

Bolgar, H., \& Fisher, L. K. (1940). The toy test: A psychodiagnostic method. Psychological Bulletin, 37, 517-518.

Bradway, K. (1981). A woman's individuation through sandplay. In K. Bradway et al. Sandplay studies: Origins, theory and practice (pp. 133-56). San Francisco: C. G. Jung Institute.

Bradway, K. (1992). Sandplay in preparing to Die. Journal of Sandplay Therapy, STA, 2(1), 13 -37.

Bradway, K. (1994). Sandplay of home and career women: Initial and final scenes. Journal of Sandplay Therapy, STA, 4(2), 36-45.

Bradway, K. (1997). Sandplay - Silent workshop of the psyche. London: Routledge.

Bühler, C. (1951). The world test: A projective technique. Journal of Child Psychiatry, 2,423 .

Crable, P. G. (1976). Women and self: An initial investigation of the feminine essence using sandplay (Doctoral dissertation, United States International University). Dissertation Abstracts International, 37(3-B), 1483-B. 


\section{Aicil Franco e Elizabeth Batista Pinto}

Denkers, G. C. (1985). An investigation of the diagnostic potential of sandplay utilizing Linn Jones' Developmental Scoring System. Doctoral dissertation, Psychological Studies Institute, Pacific Grove Graduate School of Professional Psychology, Berkeley, CA.

Franco, A. (1998). Câncer de mama: Cenários na areia antecipando a consciência. Trabalho apresentado no 3o Congresso Brasileiro de Psico-Oncologia, Goiânia, GO.

Gimenez, P. D. (1998). Orientação profissional - Uma abordagem clínica junguiana: Da técnica ao ritual. Dissertação de mestrado, Instituto de Psicologia da Universidade de São Paulo, São Paulo.

Grassano, E. (1996). O valor operativo e funcional do psicodiagnóstico na clínica [Curso Pré-Congresso]. Apresentado no 2o Encontro de Técnicas de Exame Psicológico: Ensino, Pesquisa e Aplicações. Instituto de Psicologia da Universidade de São Paulo, SP.

Grubbs, G. A. (1995). A comparative analysis of sandplay process of sexually abused and nonclinical children. The Arts in Psychopterapy, 22(5), 429-446.

Hall, J. A. (1983). Jung e a interpretação dos sonhos: Manual de teoria e prática (A. Cabral, trad.). São Paulo: Cultrix.

Jacoby, M. (1995). O encontro analítico: Transferência e relacionamento humano (C. Gerpe, trad.). São Paulo: Cultrix.

Jones, L. E. (1986). The development of structure in the world of expression: A cognitive- developmental analysis of children's sand worlds. Doctoral dissertation, Pacific Graduate Scholl of Psychology, Menli Park, CA.

Jung, C. G. (1966). The psychology of the transference. In C. G. Jung, Collected works of C. G. Jung (2nd ed., Vol. 16). Princeton, NJ : Princeton University Press.

Jung, C. G. (1991). A dinâmica do inconsciente. In C. G. Jung, Obras completas de C. G. Jung (2a ed., Vol. 8). Petrópolis, RJ: Vozes.

Kalff, D. (1980). Sandplay: A psychotherapeutic approach to the psyche. Santa Monica, CA: Sigo Press.

Kawai, H. (1985). Introduction: On transference in sandplay therapy. In H. Kawai \& Y. Yamanaca (Eds.), Studies of sandplay therapy in Japan (Vol. 2, pp. i-xi). Tokyo: Seishin-Shoboh.

Kawai, H. (1993). Sandplay and relation. Journal of Sandplay Therapy, STA, 3 (1), 18.

Kusas, M., \& Honda, T. (1990). Sandplay productions of alcoholics. Japanese Bulletin of Art Therapy, 21, 107-116.

Laplanche, J., \& Pontalis, J. B. (1989). Vocabulário da psicanálise. São Paulo: Martins Fontes. 


\section{O Mágico Jogo de Areia em Pesquisa}

Macnofsky, S. (1996). Healing into death. Journal of Sandplay Therapy, STA 5(2), 52.

Mitchell, R. R., \& Friedman, H. (1994). Sandplay: Past, present and future. London: Routledge.

Mitchell, R. R., \& Friedman, H. (1998). Quadro de referência para análise dos cenários no Jogo de Areia. (Material em processo de elaboração - comunicação pessoal das autoras para Aicil Franco com autorização especial para tradução e uso com fins de pesquisa)

Montecchi, F. (1993). Gioccando com la sabbia: La psicoterapia con bambini e adolescenti e la sandplay therapy. Milano: Franco Angeli.

Proteja. (1999). Archetypes of Brazilian goddesses. In N. T. Baum \& B. Weinberg (Eds.), The hands of creation: Sandplay images of birth and rebirth. Trabalho apresentado no 15th Congress of the International Society for Sandplay Therapy, Canada, 2001

Ramos, D. (1999). A questão psicossomática e o jogo de areia. Trabalho apresentado no Simpósio Natureza e Simbologia do Feminino: Ensaio, Pesquisa e Arte [Filmevídeo]. São Paulo: PROTEJA. 1 cassete VHS, 60 min. color. son.

Safra, G. (1996). Pesquisa em psicanálise. Caderno de Pesquisa: Registros e Anotações, (5), 24-48.

Shaia, A. (1991). Images in the sand: The initial sand worlds of men molested as children. Doctoral Dissertation, California Institute of Integral Studies, San Francisco, CA.

Silva, R. C. (1998). A falsa dicotomia qualitativo-quantitativo: Paradigmas que informam nossas práticas de pesquisas. In G. Romanelli (Org.), Diálogos metodológicos sobre prática de pesquisa. Ribeirão Preto, SP: Legis Summa.

Simpósio Natureza e Simbologia do Feminino: Ensaio, Pesquisa e Arte. (1999). [Filmevídeo] (Projetos e Estudos em Terapia com o Jogo de Areia). São Paulo: PROTEJA. 1 cassete VHS, 60 min. color. son.

Tardivo, L. S. P. C. (1997). Análise e interpretação. In W. Trinca (Org.), Formas de investigação clínica em psicologia: Procedimento de desenhosestórias/procedimento de desenhos de família com estórias (pp. 115-156). São Paulo: Vetor.

Thompson, C. (1981). Variations on a theme by Löwenfeld: Sandplay in focus. In K. Bradway et al., Sandplay studies: Origins, theory and practice. Boston: Sigo Press.

Trinca, W. (1997). Formas de investigação clínica em psicologia: Procedimento de desenhos-estórias/procedimento de desenhos de família com estórias. São Paulo: Vetor. 


\section{Aicil Franco e Elizabeth Batista Pinto}

Universidade Mackenzie. Textos recuperados em julho de 2002: http://www.mackenzie.br/universidade/psico/grupos_pesquisa/grupos.htm

Vieira, A. G. (1998). O brinquedo simbólico como uma narrativa. Psicologia: Reflexão e Crítica, 11(2), 233-252.

Weinrib, E. L. (1983). Images of the self: The sandplay therapy process. Boston: Sigo Press.

Yoshikawa, M. L. (1999). The return of spring for an adolescent girl. Journal of Sandplay Therapy, STA, 8(2), 119.

Zimmermann, E. B. (1996). Dança meditativa e caixa de areia associadas a analise verbal como técnica facilitadora de integração de processos simbólicos. Tese de doutorado, Faculdade de Ciências Médicas, Universidade Estadual de Campinas, Campinas, SP.

Recebido em 26.08.2002 Aceito em 18.02.2003 